\title{
Unilateral High Myopia and Its Complications - A case report
}

\author{
Dr. Vanlalruati Fanai ${ }^{1}$, Dr. Laishram Usharani ${ }^{2}$, Dr. Gaining Lulu Kamei ${ }^{3}$, \\ Dr. Kasomhung Soreingam ${ }^{4}$ \\ ${ }_{1,2,3,4}$ (Department of ophthalmology, Regional institute of medical sciences, Imphal)
}

\begin{abstract}
Myopia, or nearsightedness, is the single most common human eye disease in the world. Unilateral high myopia is an uncommon condition, unlike myopia in general which is found in $15 \%$ of children by age 15 years. We describe a 15 years old female who attended our OPD with inability to see and raised intra ocular pressure of the right eye, ophthalmologic examinations was done to evaluate the anatomic structures and visual functions. On examination of the right eye, there was no perception of light, pupil was mid-dilated with no reaction to light, lens show cataractous changes, eyeball was proptosed outward and laterally, manifest divergent squint of $20^{\circ}$ was noted with ciliary staphyloma at $120^{\prime}$ clock position. Slit lamp and biometric examinations of the right eye showed polychromatic lustre and ophthalmoscopic examination shows optic atrophy with extensive choroidal sclerosis. All the structures are visible in gonioscopy which confirms an open angle. Corneal transparency and corneal thickness were normal. From the clinical and ophthalmologic findings, unilateral high myopia with optic atrophy with complicated cataract with manifest divergent squint with secondary glaucoma with ciliary staphyloma was diagnosed. The case was studied in detail as unilateral high myopia is very rare in the North-Eastern part of India. This case highlights the need of awareness as our case lost her vision at a very early age due to ignorant, negligience and late referral to an ophthalmologist.
\end{abstract}

Keywords: complicated cataract, manifest divergent squint, proptosis, secondary glaucoma, unilateral high myopia.

\section{Introduction}

The prevalence of myopia varies with age and other factors. The incidence of highly myopic eyes has been increasing, especially in Asian nations (70-80\%). These eyes frequently have degenerative changes involving the sclera, choroid, vitreous, and retina. We presented a case where the patient lost her eyesight due to unilateral high myopia. Ignorant, negligence and late referral to an ophthalmologist are the contributing factors for the lost of her vision. This case highlights the need of awareness for timely referral, diagnosis and management by an ophthalmologist. A 15 year old female attended our OPD with pain, redness and inability to see from the right eye. On examination, we found that there is no perception of light in the right eye with raised intraocular pressure.

\section{Ophthalmologic Examinations}

On ophthalmologic examinations of the right eye, there was obvious proptosis of the eyeball, middilated pupil with manifest divergent squint of $20^{\circ}$. Thereafter, various other Ocular Examinations were undertaken, the findings of the same are as follows:

1. Proptosis of the eyeball was outwards and lateral.

2. Pupil was mid-dilated, not reacting to light with manifest divergent squint of $20^{\circ}$.

3. There was a thin sclera around limbus from 9 to $30^{\prime}$ clock position, $3 \mathrm{~mm}$ away from limbus.

4 .There was a ciliary staphyloma at $120^{\prime}$ clock position.

5. Lens show cataractous changes.

6. Corneal thickness of both eye was measured by pachymetry and was found to be near normal.

7. The corneal curvature was measured by keratometry and was found to be normal (K reading: right eye-

41.50/42.00, left eye- 41.00/41.25). 


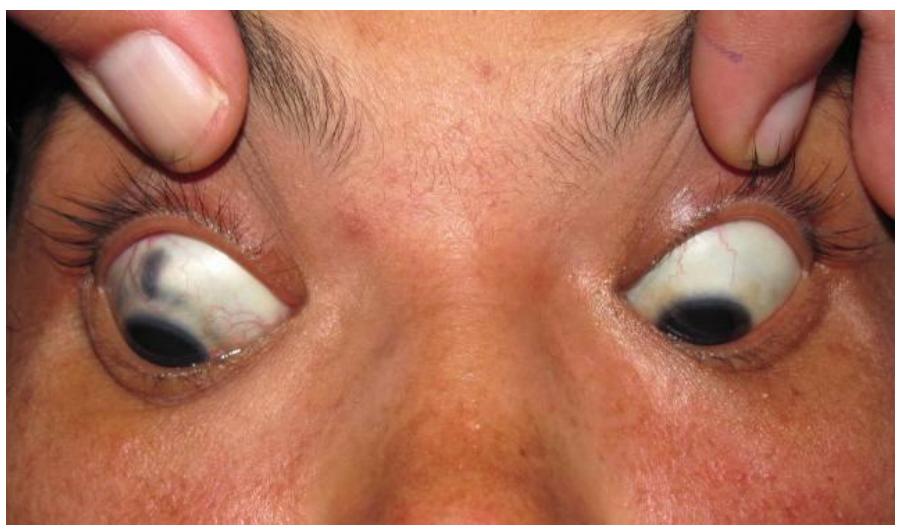

Fig.1

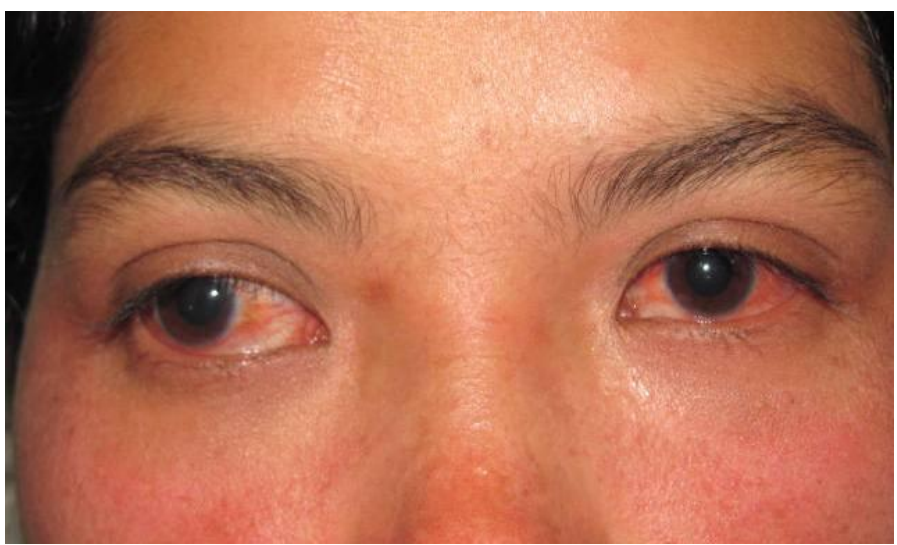

Fig. 2

\section{A - Scan Biometry:}

Biometry showed the axial length to be increased to $26.28 \mathrm{~mm}$ in the right eye compared to $23.00 \mathrm{~mm}$ in the left eye. Also the depth of the anterior chamber was increased in right eye with $4.68 \mathrm{~mm}$ compared to $3.5 \mathrm{~mm}$ in the left eye.

\section{Slit Lamp Examination:}

Pupil size was $5 \mathrm{~mm}$ in the right eye and $3.5 \mathrm{~mm}$ in the left eye i.e pupil was mid-dilated in the right eye with no reaction to light. Slit lamp examination showed clear corneas in both the eye, On examining the lens which shows cataractous changes on naked eye,we found polychromatic lustre on slit lamp examination, iris showed atrophic patches on the superior and temporal margins in the right eye. Lense and iris pattern was normal in the left eye. Gonioscopy was done in both the eyes and all structure were visible. The intraocular pressure in the right eye was $43.4 .8 \mathrm{mmHg}$ and $14.3 \mathrm{mmHg}$ in the left eye with applanation tonometry. Treatment was started for raised IOP with dorzolamide and timolol maleate $0.5 \%$ eye drop. Vision in the right eye was no perception of light and 6/18 in the left eye which was correctable to 6/6 with -1.00D . Lens and fundus appeared to be normal.

\section{Fundus examination:}

The fundus of the right eye shows optic atrophy with extansive choroidal sclerosis. The left fudus appeared to be normal. On follow up IOP was found to be reduced. Patient was continued on anti glaucoma treatment and reviewed every month.

\section{Discussions}

Myopia is a highly significant problem, not only because of its high prevalence, but also because it can contribute to visual morbidity and increase the risk for vision-threatening conditions. Because myopia is associated with reduced distance vision without optical correction, it can be a limiting factor in occupational choices. In addition, the posterior segment changes in the myopic eye place it at risk for the development of other ocular conditions .

The goals for management of the patient with myopia are clear, comfortable, efficient binocular vision and good ocular health. Effective myopia control results in less severe myopia and less vitreous chamber 
elongation than would otherwise have occurred. Regimens to reduce myopia lessen dependence on spectacles or contact lenses, but they do not lessen the risk for myopia sequelae .

\section{Various treatment modalities are :}

1)Optical correction - spectacles or contact lenses.

2) Medical - cycloplegic agents.

3) Vision therapy.

4) Orthokeratology.

5) Refractive surgery -

a)Radial keratotomy(RK)

b)Excimer laser photorefractive keratectomy(PRK)

c)Automated lamellar keratomileusis(ALK)

d)Laser in situ keratomileusis (LASIK).

\section{Conclusion}

Myopia is a vision threatening ocular disorders that need an early diagnosis and early referral to an ophthalmologists. The examination of patients who have any of the forms of myopia should include a comprehensive patient history, measurement of refraction, investigation of accommodation and vergence function, and evaluation of ocular health. The patient should be advised about available treatment options and counseled regarding the need for followup care. Vision in this patients eye could have been saved if she seek medical advice at the right time.

\section{References}

[1] Sperduto RD, Siegel D, Roberts J, et al. Prevalence of myopia in the United States. Arch Ophthalmol 1983;101:405-7.

[2] Angle J, Wissman DA. The epidemiology of myopia. Am J Epidemiol 1980;111:220-8.

[3] Curtin BJ, ed. Anisometropic myopia. In: The myopias: basic science and clinical management. Philadelphia: Harper and Row, 1985; chapter 17:449-54.

[4] Saw MS,Gazzard G,Shih-Yen EC,Chua WH. Myopia and associated pathological complications.Ophthal.Physiol.Opt.2005;25:38191.

[5] Grosvenor T. A review and a suggested classification system for myopia on the basis of age-related prevalence and age of onset. Am J Optom Physiol Opt .1987; 64:545-54. 